\title{
Der Deutsche Fassweinmarkt - Eine Warenstromanalyse der Fassweintransporte in Rheinland-Pfalz
}

\author{
Marianne Steinschulte \\ Hochschule Geisenheim, Von-Lade-Str. 1, Geisenheim, Germany
}

\begin{abstract}
In der deutschen Weinwirtschaft deckt der Fassweinhandel eine große Menge des Weinmarktes ab und nimmt somit eine herausragende Stellung ein. Für ein besseres Verständnis des komplexen deutschen Fassweinmarktes und seiner Entwicklung in den Jahren 2000 bis 2012, wurde eine explorative Datenanalyse der Fasswein-Warenströme erstellt. Die Datengrundlage bildet eine neue Datenbank der Fassweintransporte im Bundesland Rheinland-Pfalz, die zusammen mit den offiziellen Statistiken die Struktur und Entwicklung des Marktes darstellen. Die Bildung eines Zwei-Stufen-Handelsmodells zeigt deutlich die intensive Vernetzung und Arbeitsteilung sowie einen Konzentrationsprozess in der Weinbranche, zusammen mit einer Verbesserung des Qualitätsmanagements. Dagegen haben jahrgangsbedingte Ernteschwankungen keinen erkennbaren Einfluss auf die Warenströme der deutschen Fassweine.
\end{abstract}

\section{Einleitung}

Die Entwicklung des weltweiten Weinmarktes der letzten Jahre zeigt mit Eindruck die wachsende Bedeutung des Fassweinhandels [1]. Dies gilt ebenso für den deutschen Weinmarkt, wobei dieser in Deutschland eine einzigartige Struktur aufweist und für den Fassweinmarkt sehr interessante und wichtige Position einnimmt. Zum einen zeichnet sich der deutsche Weinmarkt durch einen vergleichsweise hohen Weinkonsum aus, der mit rund 20 Mio. Hektolitern (HL) nach den USA, Italien und Frankreich aktuell weltweit an vierter Stelle steht [2,3]. Zum anderen besteht in Deutschland in der Kombination aus heimischer Produktion (ca. 9 Mio. HL in 2012), einem bedeutenden Weinimport (ca. 14,9 Mio. HL in 2012) und beachtlichen Export (ca. 3,9 Mio. HL in 2012) ein für den internationalen Weinhandel wichtiger und besonderer Markt [4]. Der Handel mit Wein wird darüber hinaus begünstigt, dass der Weinmarkt weitgehend ohne Handelsbarrieren oder Steuern belegt ist [5]. Ein solch offener Markt jedoch führt $\mathrm{zu}$ einem intensiven Wettbewerb zwischen den heimischen Weinen und den importierten Produkten, vor allem im Preiseinstiegsbereich, der hauptsächlich über den Fassweinmarkt gehandelt wird.

Nach aktuellen Schätzungen werden in Deutschland über das Preiseinstiegssegment im LebensmittelEinzelhandel (LEH) bis zu 10,8 Mio. HL (davon ca. 5,7 Mio. $\mathrm{Hl}$ in den Discountern) Wein vertrieben, die damit ca. $51 \%$ der über diesen Vertriebsweg verkauften Menge ausmachen [4]. Dieser enorm hohe Anteil des beschriebenen Weinsegments zeigt deutlich die Bedeutung des Fassweinmarktes für die deutsche Weinwirtschaft. Es ist somit plausibel, dass rund ein Drittel der deutschen Weinerzeugung über Kellereien und den Lebensmitteleinzelhandel den Weg zum Verbraucher findet. Als Lieferanten und Partner des Handels sind dabei die Kellereien, die ihre Grundweine auf dem
Fassweinmarkt beziehen, darauf angewiesen, dem Handel eine kontinuierliche Lieferfähigkeit garantieren zu können. Jahrgangsbedingte Mengenschwankungen der deutschen Weinerzeugung können dadurch starke Auswirkungen auf den Markt haben. Gestalt und Intensität der Folgen sind jedoch nur äußerst schwer zu prognostizieren. Zudem werden von deutschen Handelskellereien zunehmend importierte Weine abgefüllt, die daraufhin als ReExporte in der Flasche wieder das Land verlassen [6]. Dieser Handelsstrom hat in den letzten Jahren an Bedeutung stark gewonnen und oftmals als "Drehscheibe Deutschland" bezeichnet. Dennoch ist die innere Struktur diese wichtigen Teilmarktes der Weinwirtschaft bisher kaum bekannt und nicht zuletzt aufgrund der sehr begrenzten Verfügbarkeit von statistischen Daten auch nur sehr wenige wissenschaftliche Studien durchgeführt worden. Eine neue Chance für Deutschland bietet jedoch eine neue Datenbank, die etwas tieferen Einblick in diesen Teilmarkt geben kann. Das Bundesland RheinlandPfalz nimmt für die heimische Weinproduktion eine herausragende Rolle ein, da in den dort beheimateten Anbauregionen der überwiegende Teil der heimischen Weine produziert werden (ca. $66 \%$ der gesamtdeutschen Weinproduktion) und auch einige wichtige Handelsunternehmen ihren Sitz dort haben [4,7]. Demzufolge sind die Analyseergebnisse des Fassweinmarktes in RheinlandPfalz aussagekräftig für die Entwicklung der deutschen Weine im Preiseinstiegssegment, insbesondere in Bezug auf Handelsaktivität, Substituierungseffekte und andere Einflussfaktoren.

\section{Methodik}

In der vorliegenden Studie wird die Entwicklung des deutschen Fassweinmarktes anhand der Warenströme in Rheinland-Pfalz analysiert. Als Datengrundlage hierzu dienen die Begleitscheine der Fassweintransporte aus den

This is an Open Access article distributed under the terms of the Creative Commons Attribution License 4.0, which permits unrestricted use, distribution, and reproduction in any medium, provided the original work is properly cited. 
Jahren 2000 bis 2012. Gemäß der EU-Verordnung Nr. 439 sind für alle Transporte von Fasswein, Most und Trauben die Mitführung dieser Begleitscheine verpflichtend [8]. Die Dokumente sind durchgehend nummeriert und werden von der jeweiligen weinbaukarteiführenden Landwirtschaftskammer als durchführende Dienststelle erfasst. Über das chemische Landesuntersuchungsamt erfolgt die Kontrolle der angegebenen Daten sowie die Pflege der Datenbank. Die Angaben der Begleitscheindokumente sind sehr detailliert und geben genaue Informationen über die Handelsmenge, den Absender, den Empfänger, die Qualitätsstufe, die Rebsorte und einige andere Eigenschaften. Aus Datenschutzgründen wurden die detailgenauen Angaben für diese Studie in aggregierter und anonymisierter Form zur Verfügung gestellt. Darüber hinaus werden die Handelsdaten mit den Daten der amtlichen Weinbaustatistik, wie Mostertrag, Rebfläche u.a., in Verbindung gesetzt. Für alle Qualitätsweine auf dem deutschen Weinmarkt ist eine amtliche Qualitätsweinprüfung vor Einführung in den Markt gesetzlich verpflichtend. Die Prüfungen werden durch die Landwirtschaftskammer der jeweiligen Anbauregion durchgeführt und dokumentiert ( $\$ 19$ ff WeinG). Die Daten der Anstellungen zur amtlichen Qualitätsweinprüfung wurden zusätzlich von der rheinland-pfälzischen Landwirtschaftskammer in anonymisierter und aggregiert kategorisierter Form zur Verfügung gestellt und in die Studie integriert. Zu Gunsten einer übersichtlichen Darstellung der langfristigen Entwicklung des Fassweinmarktes wurden die Daten auf Jahresebene zusammengefasst, nach verschiedenen Kategorien aufgegliedert und kombiniert.

Im Folgenden werden einige Kernaussagen der umfangreichen explorativen Datenanalyse vorgestellt. Die Ergebnisse beziehen sich rein auf die Menge der Warenströme und Organisation auf dem Fassweinmarkt ohne Berücksichtigung der finanziellen Werte und Preiseffekte. Aufgrund verschiedener zeitlicher Differenzen zwischen den dokumentierten Fassweintransporten und den Vertragsabschlüssen mit Preisverhandlungen der einzelnen Handelspartien kann ein direkter Bezug zwischen den Handelsaktivitäten und den aktuellen Fassweinpreisen nicht vorausgesetzt werden. Daher wurden alle wertmäßigen Aspekte in dieser Studie zunächst nicht mit einbezogen.

\section{Ergebnisse}

Die Entwicklung der Handelsmenge auf dem Fassweinmarkt über den betrachteten Analysezeitraum von den Jahren 2000 bis 2012, der durch die Begleitscheine dokumentiert ist, weist einen stark sinkenden Trend auf. Die auf Jahresebene aggregierten Daten zeigen darüber hinaus keine signifikanten Hinweise auf jahrgangs- und erntemengenbezogenen Einflüsse auf die Handelsaktivitäten. Der Mostertrag in dem betrachteten Analysezeitraum von 2000 bis 2012 summierte sich über alle Anbaugebiete in Rheinland-Pfalz auf jährlich ca. 6 Mio. HL Most. Mit Ausnahme des Jahrgangs 2010 war die Erntesituation in diesen Jahren mengenmäßig weitgehend gleichbleibend. Für die Erfassung der Erntemenge wurde in diese Studie der Mostertrag ausgewählt, da in der amtlich gemeldeten Weinerzeugung bereits einige Most- und

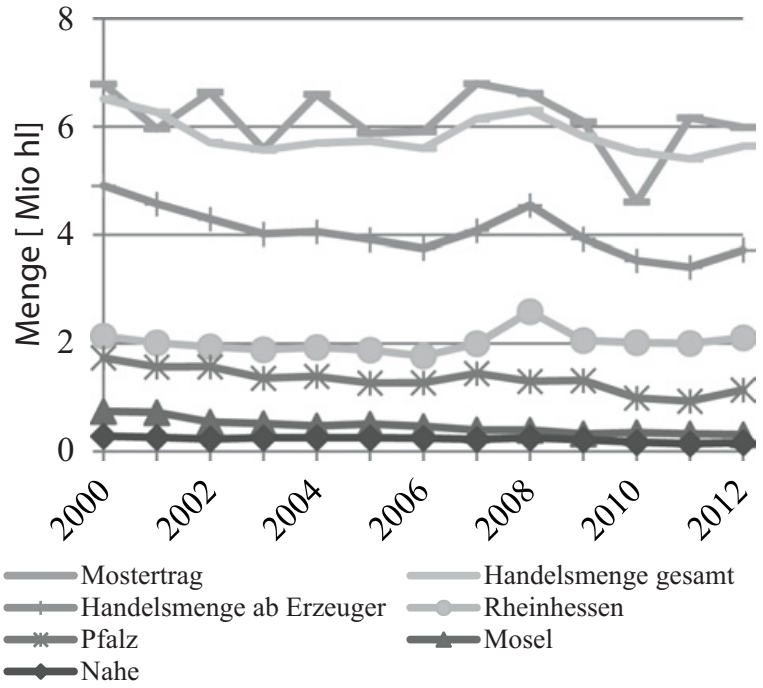

Abb. 1. Entwicklung des Fassweinangebots in den Weinanbauregionen in Rheinland-Pfalz.

Traubentransporte enthalten sind und damit das Ergebnis verzerren würde. Die anhand der amtlichen Begleitscheine erfassten Transporte weisen eine gesamte Handelsmenge aus, die in etwa der Mosterntemenge gleich kommt. Jedoch handelt es sich hierbei um die absolute Transportmenge ungeachtet möglicher Mehrfachbewegungen einzelner Partien während des Produktionsprozesses.

Um diese Redundanzen in der Handelsmenge auszuschließen, wurde diese hinsichtlich der Betriebsart der Absender gefiltert und ausschließlich Erzeugerbetriebe (Weingüter, Winzergenossenschaften, Erzeugergemeinschaften) in die weitere Analyse einbezogen (vgl. Abb. 1). Der Kurvenverlauf der Fassweintransporte zeigt zunächst geringere Schwankungen als die Erntemengen und in der langfristigen Betrachtung der aufgezeigten 13 Jahre einen signifikant sinkenden Trend. Dennoch handelt es sich hierbei um einen wichtigen Teilmarkt der deutschen Weinwirtschaft, auf dem zuletzt (in 2012) noch ca. 62\% der rheinland-pfälzischen Mosternte gehandelt wurde. Die differenzierte Betrachtung der Handelsmenge aus den einzelnen Weinanbaugebieten gibt weiter Aufschluss über deren Bedeutung und Einfluss. So wird deutlich, dass der negative Trend insbesondere durch die Pfalz beeinflusst wird, sowie auch von der Mosel. Die Handelsmengen in dem größten rheinland-pfälzischen Weinanbaugebiet Rheinhessen jedoch blieben sehr konstant (abgesehen von der Ausnahme im Jahr 2008). Diese sehr unterschiedlichen Entwicklungen der drei wichtigsten Weinanbauregionen deuten bereits auf einen Strukturwandel hin, der sich seit dem Jahr 2000 in der Branche vollzogen hat.

Der rückläufige Fassweinhandel in der Pfalz und an der Mosel hat jeweils unterschiedliche Gründe. An der Mosel hat vor allem die stark abnehmende Anbaufläche einen signifikanten Einfluss auf die niedrigeren Erntemengen und damit auch geringeren Handelsaktivitäten. Die deutliche Reduzierung der Weinbergsfläche ist jedoch in den vergangenen Jahren ein spezifisches Phänomen an der Mosel, die durch ihre geografische Beschaffenheit einen hohen Anteil an Steillagen hat, die vielfach sehr 


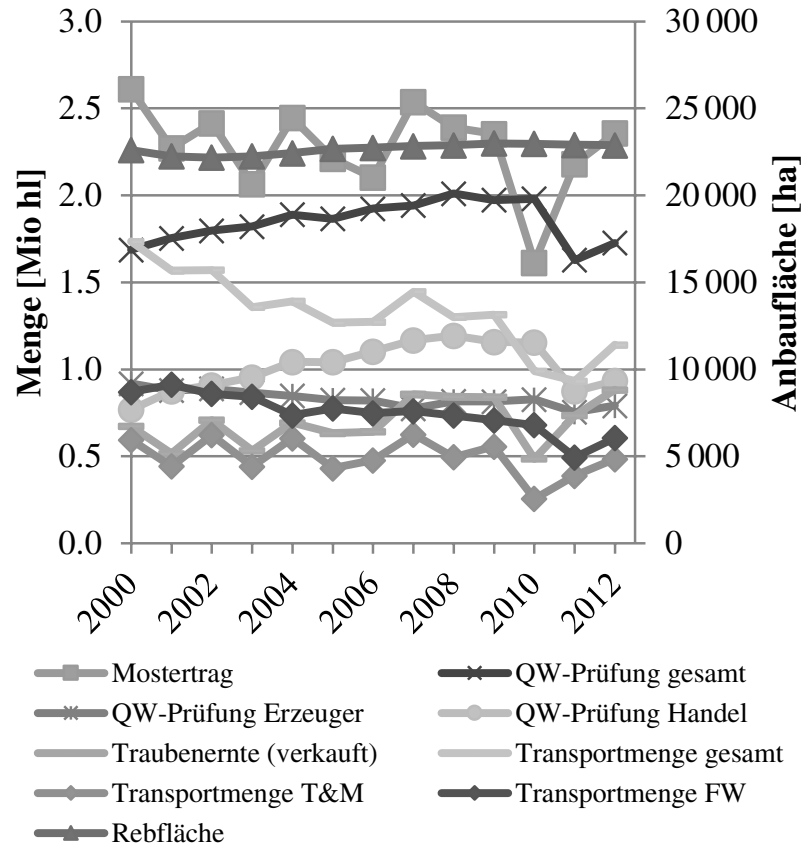

Abb. 2. Entwicklung der Fasswein- und Traubentransporte im Anbaugebiet Pfalz im Vergleich zu der Qualitätsweinmenge.

kostenintensiv in der Weinproduktion sind. Dennoch ist die Abnahme der gehandelten Fassweinmenge an der Mosel größer als die Rebflächenreduktion und muss daher noch weitere Gründe haben. Sehr deutlich wird dies in der Pfalz (vgl. Abb. 2). Während die Rebfläche und die Erntemengen, abgesehen vom Jahrgang 2010, weitgehend stabil bleiben, nimmt die transportierte Fassweinmenge stark ab und schrumpft innerhalb der betrachteten 13 Jahre um ca. 24\%. Diese Beobachtung lässt zunächst die Hypothese zu, dass die Erzeugerbetriebe ihre Ernten zunehmend behalten und als Erzeugerabfüllungen auf den Markt bringen und somit das Angebot auf dem Fassweinmarkt abnehmen lassen. Zur Überprüfung dieser Hypothese wurden die Daten der amtlichen Qualitätsweinprüfung in die Analyse einbezogen.

Insgesamt zeigt die Entwicklung der Menge der zur Qualitätsweinprüfung angestellten Weine einen positiven Trend (vgl. QW-Prüfung in Abb. 2). In der Differenzierung der Betriebsart der Ansteller jedoch wird deutlich, dass die abgefüllte Menge der Erzeugerbetriebe nicht wie vermutet gestiegen, sondern sogar leicht gesunken ist. Die zuvor stellt Hypothese der gestiegenen Erzeugerabfüllungen muss somit verworfen werden. Die Qualitätsweinmengen der Handelsbetriebe hingegen weist einen signifikant ansteigenden Trend auf. Diese Entwicklung steht zunächst im Widerspruch zu der sinkenden Transportmenge laut der amtlichen Begleitscheindokumente. Eine mögliche Erklärung dieses Paradoxons liegt in der Kategorisierung der Datengrundlage. Das entscheidende Kriterium für die Zuordnung der einzelnen Betriebe in die Kategorie Erzeuger oder Handel bezieht sich auf den Anteil der produzierten Weinmenge aus eigenen Trauben bzw. zugekauften Trauben oder Weinen. Bei sehr erfolgreichen Weingütern können es die Jahrgangsbedingungen und eine gute Nachfragesituation erforderlich machen, größere
Mengen an Weinen oder Trauben zuzukaufen, womit diese Betriebe in der Statistik in die Kategorie der Handelsbetriebe gezählt werden, obwohl sie in der Branche als Weingut wahrgenommen werden. Darüber hinaus haben einige dieser erfolgreichen Weingüter zusätzlich zu dem Weingut eine Handelsfirma mit gleicher Adresse gegründet, um einzelne Produkte des Sortiments über dieses Unternehmen vermarkten zu können. Diese werden ebenfalls statistisch als Handelsbetrieb erfasst. Auf diesem Hintergrund kann argumentiert werden, dass der sinkende Fassweinhandel bei steigenden Qualitätsweinmengen aus dem Handel in der Pfalz auf einige erfolgreiche Weingüter zurück $\mathrm{zu}$ führen ist. Aus Expertenkreisen der Branche wird dieses Phänomen insbesondere im Anbaugebiet Pfalz berichtet. Aus Gründen des Datenschutzes kann diese Entwicklung jedoch mit der bisher verfügbaren Datenbasis nicht quantifiziert und eindeutig belegt werden.

Darüber hinaus muss bei der Analyse der Begleitscheindokumente ein weiterer Aspekt bzgl. der Datenbasis beachtet werden. Für die gesetzliche Verpflichtung der Mitführung eines Begleitscheins beim Transport von Fassware gilt gemäß der EU-Verordnung Nr. 436 eine Ausnahmeregelung: für alle Transporte von unvergorenen Trauben, Most oder Maische vom Weinberg zu einem Weinkeller innerhalb eines Radius von 40 Kilometern ist die Mitführung eines Begleitscheins zwar empfohlen, jedoch nicht notwendig [8]. In seltenen Fällen und auf besonderen Antrag kann diese Ausnahmeregelung auf einen Radius von bis zu 70 Kilometern ausgedehnt werden und würde damit weite Teile der Weinanbauregionen innerhalb von Rheinland-Pfalz abdecken. Somit ist der sinkende Trend der dokumentierten Fassweintransporte auch ein möglicher Ausdruck für einen zunehmenden Traubenhandel ohne Begleitscheine. Zur Überprüfung dieser Vermutung wurden in die Analyse zusätzlich auch die Informationen aus der amtlichen Traubenerzeugungsmeldung mit einbezogen. Aus Gründen der Übersichtlichkeit ist in Abb. 2 nur die Traubenmenge aufgeführt, die auf dem freien Markt gehandelt bzw. verkauft wurde. Ebenso wurden die Transportmengen in unvergorene Mengen (Trauben und Most) und vergorene Mengen (Fasswein) differenziert. Während die verkaufte Traubenmenge bis ca. zum Jahr 2004 mit ca. 87\% zu einem konstant hohen Anteil über ein Begleitdokument verfügten und somit in der Transportdatenbank erfasst wurden, nahm dieser Anteil seither stetig ab und erreichte in 2012 eine Ratio von nur noch ca. 55\%. Damit wurden zuletzt ca. $100.000 \mathrm{~kg}$ Trauben nicht über die Transportbegleitscheine erfasst. (Anmerkung: Die Traubenerzeugungsmeldung erfolgt in der Einheit "Liter Wein" mit folgendem Umrechnungsfaktor 78 Liter Wein = $100 \mathrm{~kg}$ Trauben beziehungsweise Maische [9]) Insgesamt ist erkennbar, dass nicht nur die gehandelte Traubenmenge im Laufe des Analysezeitraums zugenommen hat, sondern vor allem der Anteil der dokumentierten Traubentransporte abgenommen hat.

Der Strukturwandel auf dem Fassweinmarkt, der sich in der Betrachtung der verschiedenen Weinanbaugebiete bereits abzeichnet, wird jedoch umso deutlicher, wenn die Empfängerregionen der Fassweintransporte in die Analyse 


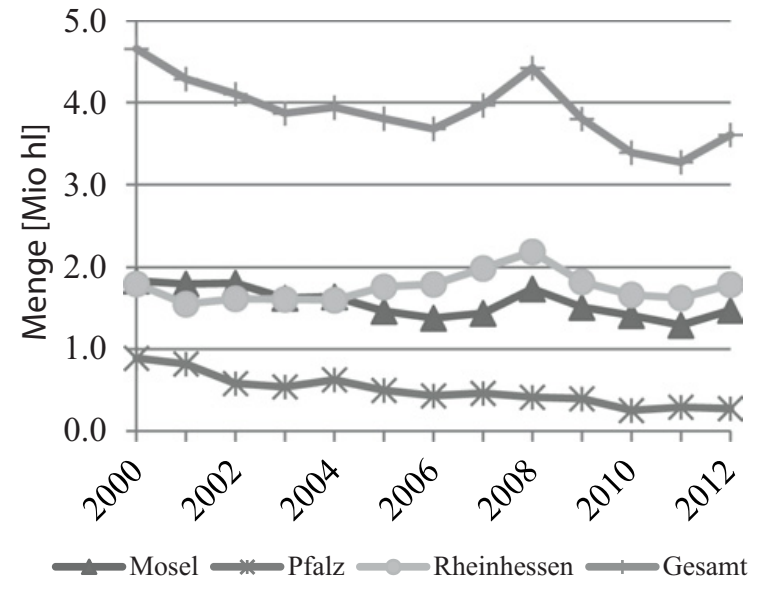

Abb. 3. Die Empfänger der Fassweintransporte der drei größten Weinbauregionen in Rheinland-Pfalz.

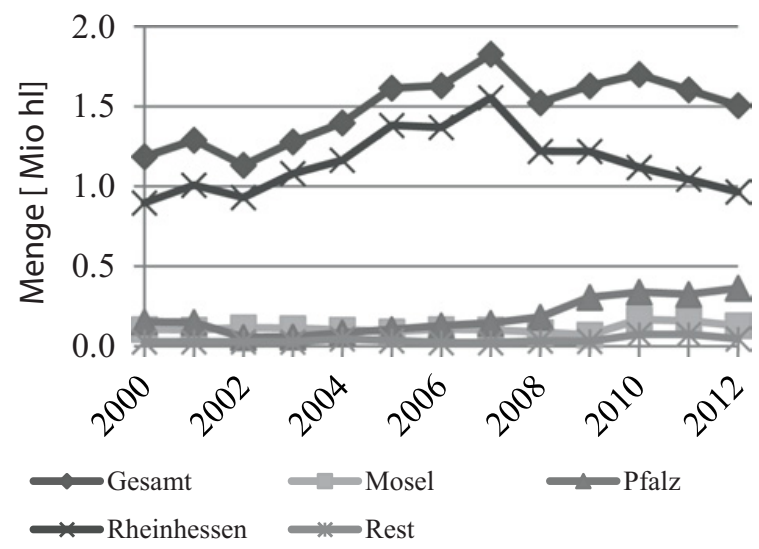

Abb. 4. Fassweinhandelsmengen zwischen den Kellereien nach Anbaugebieten der Absender.

mit eingezogen werden (vgl. Abb. 3). Das größte deutsche Weinanbaugebiet Rheinhessen tritt demnach nicht nur als wichtigste Anbieterregion auf, sondern seit dem Jahr 2004 ebenfalls als mengenmäßig stärkste Empfängerregion der Fassweintransporte direkt von den Erzeugerbetrieben kommend. Nahezu ebenso bedeutend ist die Menge der Fassweintransporte, die direkt an Unternehmen an der Mosel geliefert werden. Während in der langfristigen Entwicklung die rheinhessischen Empfänger eine weitgehend konstante Menge von ca. 1,8 Mio. Hl annahmen, ist in der Entwicklung der Handelsmenge an der Mosel wiederum ein leichter Negativ-Trend zu erkennen. Für das Anbaugebiet Pfalz ist auch hierbei eine stark sinkende Entwicklung (ca. 69\%) der empfangenen Weinmengen zu verzeichnen, die im Jahr 2012 mit ca. 300.000 HL ein deutlich unbedeutenderes Niveau erreicht haben.

In den bisher beschriebenen Analysen wurde allein die Fassweinmenge betrachtet, die von Erzeugerbetrieben versendet wurden. Diese Handelsmengen sind, wie zuvor detailliert beschreiben, rückläufig. Im Folgenden soll der Fokus auf die transportierte Fassweinmenge liegen, die zwischen den Handelsbetrieben in Rheinland-Pfalz gehandelt werden. Die Weinmenge dieses Intrahandels hat seit dem Jahr 2000 stetig zugenommen (vgl. Abb. 4). Die Entwicklung ist insofern beachtenswert, da die

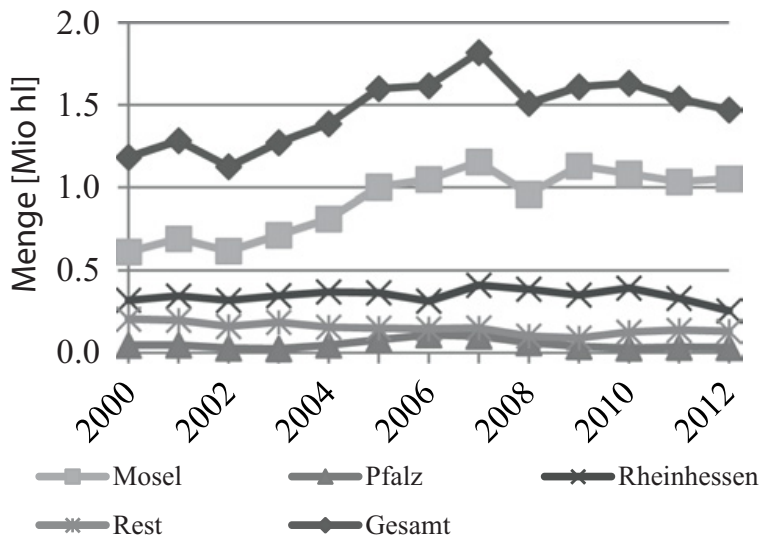

Abb. 5. Fassweinhandelsmengen zwischen den Kellereien nach Region der Empfänger.

Handelsbetriebe per Definition über keine eigene Traubenproduktion verfügen und daher zumindest die Rohware für die Weinherstellung über den Fassweinmarkt zukaufen müssen. Daher ist zu erwarten, dass diese Unternehmen hauptsächlich als Nachfrager bzw. Empfänger von Fassweintransporten auftreten. Die zunehmende Aktivität als Anbieter und Versender von Fassware ist daher ein weiterer Hinweis auf den Strukturwandel innerhalb der Branche.

Die wichtigsten Handelsunternehmen, die als Anbieter auf dem Fassweinmarkt agieren befinden sich in der Weinanbauregion Rheinhessen. Die dort gehandelte Weinmenge beläuft sich auf bis zu $85 \%$ der gesamten im Intrahandel transportierten Menge im Jahr 2007. Mit dem Jahr 2008 steigt die Intrahandels-Aktivität der Handelsunternehmen in der Pfalz an, die zuvor unbedeutend gewesen ist, während die Handelsmengen in Rheinhessen deutlich absinken. Es ist denkbar, dass es sich hierbei um eine Substituierung bzw. Verlagerung einzelner Weinpartien von den rheinhessischen Handelsbetrieben $\mathrm{zu}$ den pfälzer Unternehmen handelt. Alle weiteren Anbaugebiete in Rheinland-Pfalz treten mit nur sehr geringen Mengen als Anbieter auf dem Fassweinmarkt auf und können somit vernachlässigt werden. Hinsichtlich der Empfänger der Weinhandelstransporte aus dem Intrahandel zeigt sich eine ebenso klare Struktur: der überwiegende Teil des Intrahandels geht an Unternehmen an der Mosel, mit leicht steigender Tendenz (vgl. Abb. 5). Eine konstante und ebenfalls wichtige Rolle als Empfänger der handelsintern transportierten Weinpartien sind wiederum Unternehmen in Rheinhessen.

Die Kombination dieser beiden zunächst einzeln betrachteten Transportströme der Erzeugermengen sowie des Intrahandels macht die Entwicklung des bisher vermuteten Strukturwandels auf dem Fassweinmarkt klar ersichtlich. Unter den vielen verschiedenen Handelsbeziehungen zwischen den Betrieben der Anbaugebiete hat sich vor allem ein zweistufiger Warenstrom stark entwickelt. In der Produktion für Fassweine haben die Erzeuger in Rheinhessen die stärkste Bedeutung. Von dort werden die Weine entweder direkt an ein abfüllendes Weinhandelsunternehmen (überwiegend mit Sitz an der Mosel) transportiert oder zunächst $\mathrm{zu}$ einer weinausbauenden 
Kellerei (hauptsächlich in Rheinhessen) und von dort in einem zweiten Schritt zu den Handelskellereien an der Mosel. Dieses zweistufige Handelsmodell hat während des betrachteten Analysezeitraums stark an Bedeutung zugenommen und war in seiner Form der einzig wachsende Handelsstrom auf dem rheinland-pfälzischen Fassweinmarkt. Im jüngsten Jahr der Analyse (2012) wurden allein 0,8 Mio. HL Fasswein von den rheinhessischen Erzeugerbetrieben direkt zu Handelsunternehmen an der Mosel transportiert, weitere 0,67 Mio. HL rheinhessischer Erzeugnisse gingen an rheinhessische "Service"Kellereien, wurden dort vergoren und anschließend an die Mosel transportiert, wo die Weine abgefüllt wurden. Insgesamt betrifft dieser Warenstrom damit ca. 70\% der gesamten rheinhessischen Fassweinmenge bzw. ca. 25\% der gesamten Weinernte in Rheinland-Pfalz und hat somit einen enorme Bedeutung für den deutschen Weinmarkt erlagt.

\section{Diskussion und fazit}

Die Analyse der Fassweintransporte in Rheinland-Pfalz anhand der amtlichen Begleitscheine zeigt zunächst einen geringen Einfluss der jahrgangsbedingten Erntemengen auf die Handelsmengen auf dem Fassweinmarkt, die offenbar durch die Lagerbestände ausgeglichen werden können. Auffälliger sind die Hinweise auf einen Strukturwandel innerhalb der Branche mit unterschiedlichen Ausprägungen in den einzelnen Weinanbaugebieten. Insbesondere die Entwicklung in der Pfalz zeigt einen zunächst paradoxen Verlauf, der möglicherweise Ausprägungen eines verbesserten Qualitätsmanagements und einem Wachstum erfolgreicher Weingüter beschreibt. Darüber hinaus hat in dem betrachteten Zeitraum vom Jahr 2000 bis 2012 eine deutliche Konzentration der Warenströme innerhalb des Produktionsprozesses und der Anbaugebiete, v.a. Rheinhessen und Mosel, stattgefunden. Federführend in der Ausbildung dieser Tendenz sind einige wenige große Handelskellereien [7]. Auf diesem Hintergrund ist die Spezialisierung der führenden Unternehmen auch ein Ausdruck eines erfolgreichen strategischen Managements im gemeinsamen Wettbewerb. Während sich sogenannte "Service"-Kellereien auf die Weinproduktion konzentrieren sind andere Handelskellereien stärker in der Abfüllung und Vermarktung aktiv. Die Bildung des zweistufigen Warenstrom-Modells sowie die Position dieser "Service-Kellereien" auf dem Fassweinmarkt stehen in einem plausiblen Zusammenhang mit der Annahme erhöhter Traubentransporte (ohne Begleitdokumente). Einerseits mag diese Entwicklung in den ökonomischen Vorteilen liegen, die Arbeitsteilung und Spezialisierung hervorbringen sowie in dem Kostenvorteil durch die Nutzung von Skaleneffekten (Economies of Scale). Andererseits sind auch önologische und damit branchenspezifische Gründe bzgl. der Weinqualität möglich. Die Weine des Fassweinmarktes hatten in der Vergangenheit eine sehr heterogene Qualität je nach Jahrgang und Weinherstellungsprozess, die das Image der Weine im Preiseinstiegsbereich oftmals negativ beeinflussten. Eine kontinuierlich gute Qualität und saubere Weine kann insbesondere dann beeinflusst werden, wenn der gesamte Weinherstellungsprozess, angefangen vom Rohprodukt
Traube, kontrolliert wird. Aus diesem Grund gehen Weingüter wie Kellereien zunehmend dazu über, Trauben anstatt Weine zu kaufen und die Weinbereitung selber durchzuführen, unter Anwendung von neuen technischen Verfahren sowie bestem fachlichen Wissen. Die Integrierung sämtlicher Produktionsprozesse mag jedoch zu logistischen Herausforderungen führen, da es sich vor allem bei den Handelskellereien oftmals um große Volumina handelt. Die Spezialisierung der Betriebe kann somit auch logistische Vorteile bringen. Die Konzentrierung der Handelsunternehmen an der Mosel auf die Weinabfüllung ist zudem kompatibel mit der weltweiten Beobachtung der Weinabfüllung von Fassweinmärkten in den Zielmärkten [1].

Ursächlich beteiligt an der Verbesserung des Qualitätsmanagements und somit auch der Produktqualität im Preiseinstiegsbereich ist vermutlich auch der Lebensmittelhandel, darunter auch die Discounter, als wichtigste Kunde der Handelskellereien. Der Weinverkauf über diesen Vertriebskanal hat einen großen Anteil am Weinabsatz in Deutschland [4]. Die Verbesserungsbemühungen im Bereich der Weinqualität in diesem Marktsegment haben somit einen positiven Effekt auf das Image deutscher Weine insgesamt. Dieser Zusammenhang zwischen dem Lebensmitteleinzelhandel und dem Strukturwandel auf dem Fassweinmarkt ist plausibel und deckt sich mit den Einschätzungen von Experten der Branche. Dennoch ist eine statistische Überprüfung dieser Kausalität aufgrund fehlenden Datenmaterials bisher nicht möglich.

Die Ergebnisse dieser explorativen Studie zum rheinland-pfälzischen Fassweinmarkt entsprechen den Beobachtungen der Entwicklung des internationalen Weinmarktes, auf dem eine zunehmende Bedeutung des Fassweinhandels festgestellt wird, der vor allem durch Veränderungen der Nachfrage durch die Konsumenten aber auch von der Ausbildung neuer organisatorischer Strukturen zugunsten von Kostenreduzierung und ökologischer Nachhaltigkeit [1]. Zur Unterstützung der Ergebnisse dieser explorativen Analyse des Fassweinmarktes wird die Studie derzeit um eine qualitative Befragung ergänzt sowie statistische Zeitreihenanalysen durchgeführt.

\section{Literaturhinweise}

[1] A. Mariani, E. Pomarici, V. Boatto, The international wine trade: Recent trends and critical issues, Wine Economics and Policy, 1, 24-40 (2012)

[2] K. Anderson, S. Nelgen, Global Wine Markets 1961 to 2009, Adelaide, University of Adelaide Press (2011)

[3] K. Anderson, The World's Wine Markets, Cheltenham, UK,, Edward Elgar Publishing (2004)

[4] Deutsches Weininstitut, Deutscher Wein, Statistik 2013/2014, Mainz (2013)

[5] Europäische Gemeinschaft, Richtlinie 92/84/EWG, Amtsblatt Nr. L 316 (1992)

[6] K. Rheinschmidt, Deutsche Weinexporte 2013 - mehr Umsatz mit weniger Menge, Dienstleistungszentrum Ländlicher Raum Rheinland-Pfalz (2014)

[7] Weinwirtschaft, Die Top 100 der Weinbranche Deutsche Kellereien, Weinwirtschaft, 7, 164 (2014) 
[8] Europäische Gemeinschaft, Verordnung (EG) $N r$. 439/2009 der Kommission vom 26.05.09, Abl. L128/15 (2009)

[9] Landwirtschaftskammer Rheinland-Pfalz, Traubenernte- und Weinerzeugungsmeldung, Bad Kreuznach (2013)
[10] Statistisches Bundesamt, Weinstatistik, Ernteerhebung und Erhebung der Weinerzeugung, Qualitätsbericht, Wiesbaden (2013)

[11] D. Hoffmann, Struktur und Entwicklung des Weinmarktes in Deutschland - Außenhandel, Geisenheim (2014) 